\title{
AN EVALUATION OF THE USEFULNESS OF STROKE INDEX VALUES IN THE SWIMMING TRAINING OF PEOPLE WITH DISABILITIES
}

\author{
WOJCIECH SEIDEL ${ }^{1}$, ANDRZEJ KLAROWICZ², MAREK REJMAN ${ }^{2}$, STEFAN SZCZEPAN $^{2}$
}

\author{
University School of Physical Education in Wrocław, \\ Faculty of Sports Science, Chair of Paralympic Sports ${ }^{1}$, \\ Faculty of Physical Education, Chair of Swimming ${ }^{2}$
}

\author{
Mailing address: Wojciech Seidel, University School of Physical Education in Wrocław, \\ Chair of Paralympic Sports, 25 Witelona Street, 51-617 Wrocław, tel.: +48 71 3473128, \\ fax: +48 71 3473450, e-mail: wojciech.seidel@awf.wroc.pl
}

\begin{abstract}
Introduction. When evaluating the swimming technique of people with disabilities, a particularly important factor, besides physiological aspects, is the efficiency of the effort expended. This suggests that assessing and monitoring the effectiveness of swimming should be a regular part of training for swimmers with disabilities. Therefore, it seems important to distinguish how changes occur in the parameters that determine the effectiveness of swimming. This is especially true of anaerobic lactic exercise as the lactic acid concentration in the blood increases significantly. The aim of this study was to evaluate the usefulness of calculating velocity and the stroke index in the swimming training of people with disabilities, along with the progressive fatigue of a high-intensity interval training workout. Material and methods. The sample comprised 12 elite competitors with a disability. The experiment consisted in swimming sequential distances of $48 \mathrm{~m}, 50 \mathrm{~m}, 52 \mathrm{~m}$, and $54 \mathrm{~m}$ at maximum intensity. Competitors performed four sets of four repetitions with a 75-second interval between repetitions and 15 minutes of active resting between sets. All sets were recorded using five digital cameras with a frequency of 50 frames per second. The recorded material was analysed with the use of motion analysis software, and the stroke index was calculated. Results. There was found to be no significant change in the average swimming velocity during each set and corresponding repetition, which means that the participants were able to tolerate the training intensity. Also, the stroke index did not change to a statistically significant degree in either of the subsequent sets or the subsequent repetitions $(\mathrm{p}<0.05)$. Conclusions. We conclude that analysing the value of the swimming stroke index for people with disabilities can be a diagnostic method for assessing the effectiveness of high-intensity interval training.
\end{abstract}

Key words: disability, swimming, stroke index, high-intensity interval training

\section{Introduction}

For many years, the concept of disability did not fit within the framework of the aims and objectives of sport. Sports facilities were often not available to the disabled, mainly due to ignorance about their needs and capabilities. However, the last decade of the twentieth century saw a rise in the professional development of sport among people with disabilities. Since then, research which defines the functional capabilities of disabled people and improves training processes has moved far beyond its original rehabilitative goals [1].

Similar trends can be observed in swimming as a sports activity for people with disabilities, both in Poland and all over the world. It is no longer possible to say that swimming is only a form of rehabilitation for the disabled; on the contrary, it is increasingly becoming a professional activity. To achieve their maximum potential, athletes with disabilities must train in the same way as able-bodied competitors. The continuous improvement in achievements results in the need to seek alternative methods, because very often minor details are decisive in attaining the best results $[2,3,4]$.

Changes in the model of competitive swimming for people with disabilities mean that there is a need to seek new, more advanced training solutions that will increase the chance of achieving greater results. Success in swimming for people with disabilities increasingly depends on a precise, even model-like framework for designing training programmes, with a particular emphasis on the energy processes that occur along with the maintenance of optimal swimming technique. Consequently, it is necessary to acquire the knowledge needed to plan training loads while taking into account energy consumption, in addition to the type and magnitude of the dysfunction which impacts on the functional abilities of the competitor and is directly correlated with the optimal swimming technique $[5,6,7]$.

Speed in swimming is the ability to cover a certain distance in the shortest possible time. Speed levels are defined by three components: reaction time, the time of execution of basic movements, and the frequency of the movements. In swimming, speed is one of the most important components when performing motor skills, since obtaining the best result is only possible when the swimmer is able to control the course of the movement so that the movement is as effective as possible, despite the influence of progressive fatigue. Ineffective movements do not allow for the full use of the swimmer's energy and mental and intellectual potential; this potential is often squandered and wasted. Thus, both studies on the physiology of swimmers with 
disabilities and studies related to the biomechanical aspects of swimming present coaches and swimmers with new opportunities of finding hidden reserves. It seems reasonable, however, that aspects of the physiology of effort as well as the biomechanics of swimming should be addressed at the same time $[3,7,8]$.

Analysis of swimming technique is based on an interpretation of indexes obtained from the measurement of kinematic parameters, such as stroke length (SL), stroke repetition (SR), velocity $(\mathrm{V})$, and the stroke index (SI). Stroke frequency (SR [the cycle/s]) is one complete motor cycle of one arm over a given unit of time or the quotient of the number of cycles divided by the time it took for their completion. Stroke length (SL [m]) is the distance that a swimmer covers during one swimming cycle. Velocity $(\mathrm{V})$ is the distance covered per unit of time. The stroke index $\left(\mathrm{SI}\left[\mathrm{m}^{2} / \mathrm{s}\right]\right)$ is the product of velocity $(\mathrm{V})$ and stroke length (SL) $[9,10,11,12,13]$ and makes it possible to assess the effectiveness of the movement. Stroke index analysis results could be used by coaches to assess swimmers' progression regarding changes in swimming technique, as well as to verify the effectiveness of training methods in the context of the training cycle $[2,9,11,12,14]$.

Swimmers' test results indicate that each competitor swims with a unique individual combination of frequency and stroke length, which varies depending on the speed and the distance covered. It was noted that an increase in swimming speed is possible by increasing the number of cycles performed and shortening the distance swum in one cycle. The best swimmers are able to control and vary these parameters $[2,8,11,12,13]$.

The above considerations seem to be particularly important for people with disabilities, primarily due to their limited functional capabilities which consequently affect their ability to create a drive force. The key, therefore, seems to be to find an optimal swimming technique that makes it possible for the swimmers to select their stroke rate (SR) and stroke length (SL), which will allow for the optimal use of their functional capabilities $[2,3,4,15]$.

It is generally agreed that the threshold considered for aerobic transformation corresponds to a concentration of $2 \mathrm{mmol} / \mathrm{l}$ lactic acid in the blood, while the anaerobic threshold is approximately $4 \mathrm{mmol} / \mathrm{l}$. The level of lactic acid in the blood while resting is close to $1 \mathrm{mmol} / \mathrm{l}$. It can be assumed that in addition to physiological aspects, another important factor for swimmers with disabilities is the efficiency of their effort, which is directly linked to swimming technique. This implies that fixing the anaerobic transformation threshold at the level of $4 \mathrm{mmol} / \mathrm{l}$ simply indicates the value at which aerobic and anaerobic exercises are distinguished. However, this does not indicate the true potential of the optimum technique when performing the movement, which is closely associated with the efficiency of the effort of each competitor. Hence, monitoring and evaluating the swimming stroke should be a standard element of the technical training of swimmers with various dysfunctions while taking into account variations in the level of their swimming ability. Therefore, it seems important to identify the changes in the parameters when determining the effectiveness of the movements performed. This is especially true of anaerobic lactic exercise, during which the concentration of lactic acid in the blood increases significantly. It is desirable for swimmers to maintain the correct technique when performing subsequent repetitions over the distance at maximum intensity.

As a result of such an analysis, it will be possible to determine the maximum swimming distance of an individual. The competitor will then be able to perform the effort several times at maximum intensity while preventing a decline in the stroke index. It is assumed that if the swimming distance is too long or the competitor is unable to withstand successive repetitions at the same speed, the stroke index will decrease.

The aim of the present study is to evaluate the usefulness of calculating velocity (V) and the stroke index (SI) in the swimming training of people with disabilities, along with the progressive fatigue brought about by a high-intensity interval training workout. For this reason, the research hypothesis was that analysis of the value of kinematic indicators (V and SI) can be a useful and diagnostic method for evaluating the effectiveness of training and provide insight into the development of highintensity interval training programmes.

\section{Material and methods}

\section{Subjects}

Twelve highly trained male swimmers with disabilities (16.3 \pm 2.7 years of age, $1.80 \pm 4.8 \mathrm{~m}$ in height, $775 \pm 2.8 \mathrm{~kg}$ in weight, experience of $7 \pm 2$ years) took part in the study. The swimmers were characterised by functional capabilities (with swimming classes of S9-S10) and all were elite competitors - each was a medallist in the Polish Cup, the Polish Cup for juniors, or even more prestigious competitions. The swimmers trained on a regular basis, taking part in 10 training sessions a week. Both the swimmers and their parents were informed of all the conditions of the research. Each participant gave written consent to take part in the experiment. The ethics committee accepted the grounds for and means of conducting the tests.

\section{Procedure}

The experiment was carried out after two days of rest. All the swimmers were well-nourished and hydrated. None of the participants claimed to be injured. Testing was conducted in a 25-m swimming pool. Prior to the experiment, the swimmers performed a standard warm-up (swimming the distance of 600$800 \mathrm{~m}$ ), after which each swimmer rested until reaching the subjective state of readiness for performing effort at maximum intensity. The starts were performed from a stationary lying position, and hence there was no push-off from the walls. The swimmers' legs were held by the Assistant Trainer.

After the signal, without pushing-off from the wall, the competitors swam using a crawl stroke at the maximum possible intensity over the following distances: $48 \mathrm{~m}, 50 \mathrm{~m}, 52 \mathrm{~m}$, and 54 $\mathrm{m}$. This means that the volume of the training load increased during each of the series. Competitors performed four sets of four repetitions with a 75 -second interval between repetitions with 15 minutes of active rest between sets. All sets were recorded by five digital video cameras with a frequency of 50 frames per second. The cameras were positioned along the pool at 5-m intervals. Prior to the experiment, the middle of the lane was equipped with a 25-m calibration line with plastic balls placed along the line at $1-\mathrm{m}$ intervals. The line was then recorded to be used as a reference point and removed from the swimming lane. The participants wore swimming caps which had two circular calibration marks on both sides with a diameter of $7 \mathrm{~cm}$.

\section{Measurements}

The recorded material was analysed with the use of motion analysis software. The analysis consisted in registering the head position of the swimmer at the beginning and the end of each swimming cycle. On these grounds, the average velocity and the stroke index were calculated. 


\section{Statistical analysis}

Empirical distributions of the average velocity and the stroke index did not differ substantially from the normal distribution, as proved by applying the Shapiro-Wilk test. The comparison between average velocity values and the stroke index in specific sets was conducted using the Student's t-test to obtain dependent samples.

\section{Results}

Average velocity was calculated as the quotient of the distance of a swim and its duration. In the first 3 training sets, the participants covered distances from $48 \mathrm{~m}$ to $54 \mathrm{~m}$ at a similar velocity. However, in the final training set, the average velocity decreased slightly (tab. 1). Nevertheless, the observed changes were small and statistically insignificant $(\mathrm{p}<0.05)$.

Table 1. Average swimming velocity in each training set

\begin{tabular}{|c|c|c|c|}
\hline Set & Distance & Velocity ( \pm 1 SD) & $p$ \\
\hline \multirow{4}{*}{ Set 1 } & $48-50$ & $1.305( \pm 0.170)-1.295( \pm 0.163)$ & 0.080 \\
\cline { 2 - 4 } & $50-52$ & $1.295( \pm 0.163)-1.262( \pm 0.162)$ & 0.619 \\
\cline { 2 - 4 } & $52-54$ & $1.262( \pm 0.162)-1.264( \pm 0.165)$ & 0.977 \\
\hline \multirow{3}{*}{ Set 2 } & $48-50$ & $1.269( \pm 0.191)-1.299( \pm 0.193)$ & 0.705 \\
\cline { 2 - 4 } & $50-52$ & $1.299( \pm 0.193)-1.259( \pm 0.176)$ & 0.601 \\
\cline { 2 - 4 } & $52-54$ & $1.259( \pm 0.176)-1.265( \pm 0.176)$ & 0.931 \\
\hline \multirow{3}{*}{ Set 3 } & $48-50$ & $1.266( \pm 0.164)-1.282( \pm 0.180)$ & 0.031 \\
\cline { 2 - 4 } & $50-52$ & $1.282( \pm 0.180)-1.241( \pm 0.184)$ & 0.574 \\
\cline { 2 - 4 } & $52-54$ & $1.241( \pm 0.184)-1.257( \pm 0.190)$ & 0.828 \\
\hline \multirow{3}{*}{ Set 4 } & $48-50$ & $1.166( \pm 0.083)-1.173( \pm 0.094)$ & 0.361 \\
\cline { 2 - 4 } & $50-52$ & $1.173( \pm 0.094)-1.163( \pm 0.124)$ & 0.841 \\
\cline { 2 - 4 } & $52-54$ & $1.163( \pm 0.124)-1.158( \pm 0.109)$ & 0.919 \\
\hline
\end{tabular}

Slightly larger variations were noticed in the case of the stroke index, which was calculated as the quotient of the stroke length (SL) and the average velocity (V). Changes in the value of the stroke index are illustrated in figure 1 . A higher stroke index value indicates increased swimming efficiency, which in turn suggests a more productive technique and a greater efficiency of effort.

In the first training set, the stroke index was the highest in the first and last repetition, whereas the lowest index was recorded during the second repetition over a distance of $50 \mathrm{~m}$. In the second set, the stroke index rose as the distance increased, which indicates that the maximum efficiency of swimming occurred in the last repetition - over the 54-m distance. In the third and fourth training sets, the stroke index decreased quite significantly; however, it grew in the subsequent repetitions of the aforementioned sets, that is as the distance increased (fig. 1). However, none of the changes in the value of the stroke index, neither in the subsequent repetitions nor sets, were statistically significant $(\mathrm{p}<0.05)$.

\section{Discussion}

In the swimming of people with disabilities, it is common practice to use training methods based on models designed for able-bodied swimmers. The results achieved by swimmers with disabilities continue to improve. Due to increased competition and the desire to maximise results, swimmers with disabilities are treated as competitive athletes [1].

It is known that the intensity of the training load and the swimmers' corresponding heart rate values during the workout must not exceed the threshold. In the case of able-bodied swimmers, these values are determined on the basis of age, competitive experience, sports ability, and the period of the training cycle. Additionally, for people with disabilities, factors requiring consideration are the nature and magnitude of the swimmer's dysfunction [16]. In the present study, certain values which seem to be optimal were assumed; however, the practical application of these values does not guarantee that the desired effect will be attained. Coaches of swimmers with disabilities face a much more difficult and complex problem when planning and implementing training programmes than coaches of able-bodied swimmers. It is impossible to develop a universal training model for swimmers at a given point of their career, even only for one type of dysfunction. An appropriate, and above all, efficient selection of the correct training load as well as the accurate

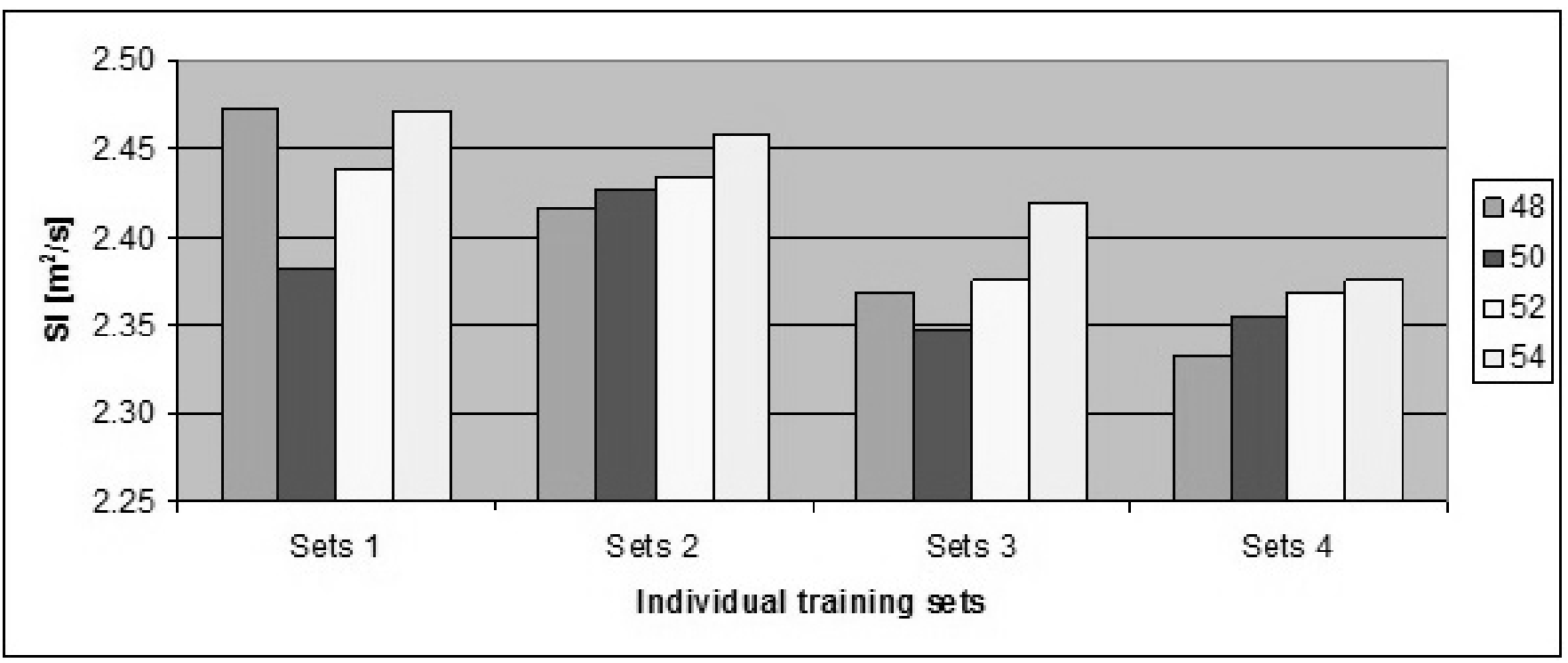

Figure 1. The swimming stroke index in each training set 
identification of the required intensity for a given effort requires significant experience as well as relevant cyclical research in this field. When selecting the load and training type, it is necessary to determine (individually for each swimmer) the intensity of the effort at which the transition from the aerobic to anaerobic exercise occurs $[2,4,17,18,19,20]$.

The scientific literature on this issue confirms that there is a relationship between the selection of the training load and the condition of the swimmer. Therefore, it is necessary to determine the current level of their physical condition using a series of tests and to monitor the effects of training on swimmers with disabilities in order to optimise workloads according to their individual functional ability. In this experiment, based on the recordings of the five digital video cameras set along the swimming pool, the distance and the number of completed cycles were identified. On these grounds, the average velocity and the stroke index were calculated. It seems unlikely that the method used contains significant errors in measurement accuracy or sampling frequency. However, there is a small possibility that inaccuracy could arise from swimmers pushing-off from the walls after preparing for the return swim. With the above in mind, the relative error was estimated to be around 3\% [9, 13].

The aim of this work was to search for new and more advanced training solutions that will increase the chances of competitors with disabilities obtaining better results. The research issue arose from the fact that in recent years the level of sport has increased significantly. One of the means for improving athletic performance and obtaining even higher results at competition level is to focus on the development of methods for managing and training competitors with disabilities. The above aimed at defining reliable methods for the support and design of technical training, while taking into account the intensity of the effort. It is assumed that an incorrectly selected distance in lactic training could cause a significant decrease in velocity (as a result of the change in the energy) or could lead to inefficiency when swimming over a certain distance, which, in turn, could mean that the competitors were not able to take full advantage of their potential speed, and sometimes the energy they expended was simply wasted. The research problem was resolved by establishing the mean velocity at which the competitors traversed the consecutive distances as well as through calculating the stroke index. On the basis of changes in the value of the average velocity, it was speculated that competitors would be able to continue the exercise at maximum intensity. Moreover, on the basis of changes in the value of the stroke index, it was speculated that the effort would be performed in an effective manner. Indirectly, such results would constitute evidence of the efficient expenditure of effort and correct swimming technique.

In the literature on the subject, scientific resources dedicated to this area of study were available; however, the authors were unable to find any research that could precisely confirm the causal relationship between the above factors. This issue is particularly important for swimmers with disabilities, where it is an important element in improving individual swimming technique and anaerobic capacity, especially since research has shown that the effectiveness of swimming is affected by both the duration of the training and the distance swum [2, 4, 17]. Therefore, it seems reasonable to seek individual training methods which would help in objectifying training loads while taking into account the optimal swimming technique. Also, in the case of swimmers with disabilities, the measurement of the stroke index could easily be introduced into the training programme by coaches as an active indicator for the purpose of monitoring the competitors' progression during the season $[2,19]$. The fact that there was no significant decline in the average swimming speed during each repetition indicates that the swimmers were able to tolerate the increasing concentration of lactic acid. This suggests that the distance covered by the competitors was too short to lead to a significant decrease in swimming velocity. However, the question arises as to whether, despite these new findings, there was a decrease in the effectiveness of the swimming, which in turn would signal greater energy expenditure. However, changes in the value of the stroke index in various repetitions and training sets were small and statistically insignificant $(\mathrm{p}<0.05)$. It can be concluded that changing the quantity of sets, the number of repetitions in the set, and the length of the individual distances may lead to clearer results.

Controlling the level of the competitors' condition should be an integral part of the training process if programmes are to be developed and managed effectively. Despite the fact that invasive studies (e.g. assessing the concentration of lactic acid in the blood) allow for a precise assessment of the competitors' condition, this is not a tool available for all trainers, nor is its use within the present capabilities of all trainers. In such cases, it is possible to use non-invasive methods, such as conducting swimming speed tests and analysing basic kinematic parameters $[7,10,12,13]$.

Recursive determination of the anaerobic transformation threshold is currently the primary means of determining the level of changes in the conditioning of swimmers, which can also be applied to swimmers with disabilities. It depends to a large extent on the impact of training, genetics, and, for swimmers with disabilities, the type and degree of their dysfunction. It can therefore be assumed that the above-mentioned factors, although they concern only a select area of training evaluation, form an integral part of the training monitoring system. Moreover, the research conducted in this field contributes to trainers' expertise and can be used in developing a more accurate rationale for the process of training for swimmers with various disabilities and with different levels of sports experience.

\section{Conclusions}

Analysis of the value of the swimming stroke index for people with disabilities can be a diagnostic method for assessing the effectiveness of high-intensity interval training.

Evaluating kinematic indicators for swimmers with disabilities can provide valuable information when determining the distance, the number of training sets, as well as the numbers of repetitions during high-intensity interval training programmes.

\section{Literature}

1. McCann C. (1996). Sports for the disabled: The evolution from rehabilitation to competitive sport. British Journal of Sports Medicine 30, 279-280.

2. Pelayo P., Sidney M., Moretto P., Wille F., Chollet D. (1999). Stroking parameters in top level swimmers with a disability. Medicine E Science in Sports E Exercise 31(12), 1839-1843.

3. Malone L.A., Sanders R.H., Schiltz J.H., Steadward R.D. (2001). Effects of visual impairment on stroke parameters in Paralympic swimmers. Medicine $\mathcal{E}$ Science in Sports $\mathcal{E}$ Exercise 33(12), 2098-2103.

4. Fulton S.K., Pyne D.B., Hopkins W.G., Burkett B. (2010). Training characteristics of paralympic swimmers. Journal of Strength $\mathcal{E}$ Conditioning Research 24(2), 471-478. 
5. Capelli C., Pendergast D.R., Termin B. (1998). Energetics of swimming at maximal speeds in humans. European Journal of Applied Physiology 78(5), 385-393.

6. Barbosa T.M., Lima F., Portela A., Novais D., Machado L., Colaço P. et al. (2006). Relationships between energy cost, swimming velocity and speed fluctuation in competitive swimming strokes. In J.P. Vilas-Boas, F. Alves, A. Marques (eds.), Biomechanics and medicine in swimming (pp. 192194). Porto: Portuguese Journal of Sport Science.

7. Barbosa T.M., Bragada J.A., Reis V.M., Marinho D.A., Carvalho C., Silva A.J. (2010). Energetics and biomechanics as determining factors of swimming performance: Updating the state of the art. Journal of Science and Medicine in Sport 13(2), 262-269.

8. Pelayo P., Moretto P., Robin H., Sidney M., Gerbeaux M., Latour M.G. et al. (1995). Adaptation of maximal aerobic and anaerobic tests for disabled swimmers. European Journal of Applied Physiology and Occupational Physiology 71(6), 51217.

9. Haljand R. (1997). Methods of kinematic evaluation for competition techniques in swimming and conclusions for training. In K. Daniel, U. Hoffman, J. Klauck (eds.), Kolner Schwimmsportage: Symposiumsbericht (pp. 54-61). Bockenem, Germany: Sport Fahnemann.

10. Chollet D., Pelayo P., Delaplace C., Tourny C., Sidney M. (1997). Stroking characteristic variations in the 100-m freestyle for male swimmers of differing skill. Perceptual and Motor Skills 85(1), 167-177.

11. Huot-Marchand F., Nesi X., Sidney M., Alberty M., Pelayo P. (2005). Variations of stroking parameters associated with $200 \mathrm{~m}$ competitive performance improvement in top-standard front crawl swimmers. Sports Biomechanics 4(1), 8999.
12. Aujouannet Y.A., Bonifazi M., Hintzy F., Vuillerme N., Rouard A.H. (2006). Effects of a high-intensity swim test on kinematic parameters in high-level athletes. Applied Physiology, Nutrition, and Metabolism 31(2), 150-158.

13. Toussaint H., Truijens M. (2005). Biomechanical aspects of peak performance in human swimming. Animal Biology 55(1), 17-40.

14. Toussaint H.M., Carol A., Kranenborg H., Truijens M.J. (2006). Effect of fatigue on stroking characteristics in an arms-only 100-m front-crawl race. Medicine $\mathcal{E}$ Science in Sports $\mathcal{E}$ Exercise 38(9), 1635-1642.

15. Satkunskiene D., Schega L., Kunze K., Birzinyte K., Daly D. (2005). Coordination in arm movements during crawl stroke in elite swimmers with a loco-motor disability. Human Movement Science 24(1), 54-65.

16. Daly D.J., Vanlandewijck Y. (1999). Some criteria for evaluating the 'fairness' of swimming classification. Adapted Physical Activity Quarterly 16(3), 271-289.

17. Cregan K., Bloom G.A., Reid G. (2007). Career evolution and knowledge of elite coaches of swimmers with a physical disability. Research Quarterly for Exercise and Sport 78(4), 339-50.

18. Capelli C., Zamparo P., Cigalotto A., Francescato M.P., Soule R.G., Termin B. et al. (1995). Bioenergetics and biomechanics of front crawl swimming. Journal of Applied Physiology 78(2), 674-679.

19. Dummer G., Bare J. (2001). Including swimmers with a disability: A guide for coaches. Coaches Quarterly 7(2), 18-26.

20. Zamparo P., Capelli C., Cautero M., Di Nino A. (2000). Energy cost of front-crawl swimming at supra-maximal speeds and underwater torque in young swimmers. European Journal of Applied Physiology 83, 487-491.

Submitted: September 3, 2015

Accepted: August 4, 2016 